\title{
Quantitative Analysis of the Topologic Morphology of Urban Street Networks Based on System Coupling Theory
}

\author{
Yan XIAO, School of Architecture and Fine Art, Dalian University of Technology, China \\ Bingxin WANG, School of Architecture and Fine Art, Dalian University of Technology, China \\ Hui SUN, School of Architecture and Fine Art, Dalian University of Technology, China
}

\begin{abstract}
Researchers are increasingly paying attention to urban morphology to address problems regarding urban form and to sustain the development of urban economy, society, and environments. A preliminary research framework was built to conduct coupling analyses on street form and block functions. These analyses are implemented using a planar graph method and using quantitative descriptions of the urban streets functions, but the coupling relation of street morphology and block function cannot be well defined, and it often cannot be analyzed in multi-level and multi-scale. Along with two proposed measuring parameters (connectivity and accessibility of coupling networks), the framework was used to quantitatively analyze the coupling coordination degree of the topologic morphology and functional structure of block samples for various urban streets. Through empirical research on different samples from Dalian, China, we validated the operability and urban street network coupling analysis in different spatial regions in built environments. This technique can be used to study the overall spatial morphology and design urban streets at different scales and scopes. Further, it helps recognize the space and cultural connotations of urban streets via spatial coupling, compare different urban textures, and predict design results to foster discussions on the optimization of urban planning design schemes.
\end{abstract}

\section{Keywords}

Urban street networks; topologic morphology; system coupling; quantitative analysis

\section{Introduction}

Since around 1990, sustainable development has become an influential concept. It has shaped discussions of urban form as cause of environmental problems (Bibri et al, 2017; Thomas, 2003). The street, one of the most important material carriers in urban space, plays a key role in the formation of urban forms and textures (Scellato et al, 2006). Urban street networks evolve with their built environments, given economic developments, and social and cultural conditions (Hillier, 1984; Jacobs, 1993). This is why they also have an important impact on the expansion of urban economic activities and improvements to living facilities (Hacar \& Geosciences; Hillier \& Information, 2008). Studies in urban theory moved past a focus on the aesthetic paradigm of physical form (Jacobs, 1993; Lynch, 1984; Marshall, 2004). These changes in studies and ideas reflect street vitality to different extents. Since the beginning of the $21^{\text {st }}$ century, new technologies have developed for the quantitative description of urban street morphology and their complexity (Cardillo et al, 2006). 
In China, through paradigm shifts and large-scale urbanization, quantitative research developing new perspectives on street has become an important topic (Tang et al, 2018; Wang et al, 2019; Yue \& Zhu, 2019). Street environmental behavior is now the main direction of quantitative research on street morphology. And street configuration is the basic spatial unit. Streets' geometric characteristics can be integrated into evaluations of their functional layout (Marshall et al, 2018). Using computer programs, we can measure the scene-design habitability of city streets. This has promoted the use of street designs in more detailed descriptive studies ( Chan et al, 2011; Xie \& Levinson, 2007).

Despite the verified influence of topologic morphology on street activities and vitality, the correlation of street morphology and function (of parcels and blocks) is a scientific problem that has not been addressed (Sharifi \& Environment, 2019). Through these findings, we have developed measurement indices of urban system coupling at the local scale. This is the basis for measuring street space system coupling (Lämmer et al, 2006; Salingaros, 2005). So, there is a need to develop systems of urban street space into integrated coupling systems. These systems will enable explorations of the coupling relation between streets' spatial morphologies and their functions.

We will present a theoretical framework of system coupling coordination for urban morphological characteristics and urban planning. We have conducted quantitative research on the coupling coordination functions and topologic morphologies of urban streets. We defined a multi-scale for this research, and we took full advantage of streets' morphological characteristics and topologic structure. Our aim was to examine the interactions between land use functions and social activities.

\section{New method proposal}

\subsection{Principles of coupling research on urban streets}

There is a relation of interactive coupling between streets' spatial morphology and function. Their function drives their formation and affects the evolution of their forms (Batty \& Longley, 1994). But good street morphology is the material basis and prerequisite for the normal operation of its function (Salingaros, 1998). This relation multiplies over time and space, through constant interrelationships and mutual restrictions and promotions. Our research framework allows for coupling analyses of urban streets. We base these analyses on system coupling, taking urban streets as special systems.

The convenience of urban streets is in their connections and paths. They connect across divergent functions and ease the efficient flow of matter and energy within a city. This is a necessary aspect of the formation of street vitality. Distinct functions may attract pedestrians to gather and linger.

We treat the street intersection as a network node. That is, we construct the edge of a network based on the coupling between nodes, and we connect nodes with distinct functions by their edges. In this way, we can set up a coupling analysis model for an urban street network. The edges joined at street intersections reflect the attraction of spatial entities and their diverse functional attributes. Individuals' chances to walk in these spaces, like their potential to interact at gatherings and activities, belongs at the nodes. More connected areas can increase the overlap of social opportunities. And the daily activities of pedestrians and residents converge their paths. We will build new parameters for quantitative analyses based on connectivity and accessibility.

Based on typical network density and intersection arrangement in China, we determined unit moduli for a network of streets in their spatial grid. With $300 \mathrm{~m}$ as the basic modulus, we used 2-time and 4-time moduli as the expansion moduli. And we used moduli of 300,600, and 1,200 $\mathrm{m}$ for this discussion of the coupling effect of urban streets at different scales. Urban streets' functional hierarchy of morphology and function requires multi-level and multi-scale description methods in the following quantitative analyses. 
As we measured parameters in the process, we performed a longitudinal analysis at different levels of scale. We also conducted a transverse analysis, focused on different land uses for streets and blocks. These included residential $(R)$, administration and public services $(A)$, commercial and business facilities $(B)$, and green spaces and public squares $(G)$, etc. This was according to the Code for Classification of Urban Land Use and Planning Standards of Development Land (GB 50137-2011).

\subsection{System coupling analysis technology for urban street space}

We integrated the ArcGIS10 and Matlab2008 platforms. In this way, we extracted and pre-treated street space information. We also stored and established a database, modeled operations, analyzed spatial data, and displayed a graphical representation of the results from our analysis.

Data extraction for urban street topologic morphology. We followed land use standards for legend colors in the classification of urban land use in China to process a land use map. We used image gray-scale processing and concentration analysis. In this way, we extracted gray-scale images of grids and their functional attributes. Contraposition superposition of integrated data on urban street morphology and function at a constant scale can reflect changes in the distribution characteristics of urban streets' functions and quantities within a given area. The nodes fall into the image grids of different gray values and attributes. The distance from one node to another is also calculable according to the information from these grid units.

Quantitative description tool chart for urban streets. We will discuss the statistics of node connections for walking accessibility. This shows up in different functions and scales in a coupling network. We will look at it within pedestrians' walking time limits. That is, we will examine within a radius of 20 minutes' walk (about 1,200 m); (Stratford et al, 2020). This applies via the coupling network generated by the edges between two nodes of different gray attributes, in the grid image of the street functions.

\subsection{Measuring index system for the system coupling of urban street space}

As listed in Table 1, our measuring parameters included the coupling clustering coefficient (CC) and average coupling clustering coefficient ( $A C C$ ). We used these to describe the connectivity of street space nodes, and nodes' influence in the coupling network. This first group of parameters reflects the importance and accumulation of nodes in the coupling network (Gastner et al, 2006). The second group of measuring parameters consisted of average coupling path length $\left(A L_{p}\right)$ and total coupling path length $\left(L_{p}\right)$. We used these to describe node accessibility within local and global ranges. They reflect the coupling accessibility of the coupling network (Ravasz \& Barabási, 2003). These two groups of measuring parameters can describe well-matched spatial components of streets. These aid in decisionmaking for symbiosis and evolution trends of morphology and function (Jiang et al, 2004).

Table 1. Measuring parameters and types.

\begin{tabular}{|c|c|c|}
\hline Parameter variable & Description information & Description type \\
\hline Coupling clustering coefficient & $\begin{array}{l}\text { The ratio of the actual number of edges } \\
\text { to the maximum number of edges }\end{array}$ & Connectivity of the \\
\hline $\begin{array}{l}\text { Average coupling clustering } \\
\text { coefficient }\end{array}$ & $\begin{array}{l}\text { Average value of clustering coefficients } \\
\text { of all nodes }\end{array}$ & coupling network \\
\hline Total coupling path length & All the length values of the coupled Path & Accessibility of the \\
\hline Average coupling path length & $\begin{array}{l}\text { The average length of all the coupled } \\
\text { paths }\end{array}$ & coupling network \\
\hline
\end{tabular}


Quantitative settings of the coupling measuring index: Coupling clustering coefficient. The coupling clustering coefficient is the ratio of the actual number of connected edges that a node has with its neighbors to the maximum number, of possible connected edges, that a node has with its neighbors. It can be written as:

$$
C C=\sum_{i} \frac{k_{i}}{N}
$$

$C C$ is the coupling clustering coefficient of node $i, N$ is the actual number of connected edges in the coupling network, and $k_{i}$ is the actual number of connected edges that node $i$ has with its neighbors within distance of its modulus.

Quantitative settings of the coupling measuring index: Average coupling clustering coefficient. The average clustering coefficient is the average value of the clustering coefficients for all nodes. The higher the value, the higher the coupling connectivity degree of the coupling network, and the closer the connection between nodes. It can be written as:

$$
A C C=\frac{\ln 2 N}{\ln (n \cdot(n-1))}
$$

ACC is the coupling clustering coefficient of the whole network, $N$ is the actual number of connected edges in the coupling network, and $n$ is the number of nodes in the coupling network.

Quantitative settings of the coupling measuring index: Total coupling path length. Total coupling path length is the length of the path between any two nodes in a network. This characterizes the accessibility degree of a spatial coupling network.

$$
L_{P}=\sum_{i, j} d\left(v_{i}, v_{j}\right)
$$

$L_{p}$ is the total length of coupling path in the network; $d\left(v_{i}, v_{j}\right)$ is the distance of node $i$ and node $j$.

Quantitative settings of the coupling measuring index: Average coupling path length. Average path length shows the average level of accessibility for a coupling network. It distinguishes a spatial coupling network from one which is inaccessible and inefficient.

$$
A L_{P}=\frac{2}{n \cdot(n-1)} \cdot \sum_{i, j} d\left(v_{i}, v_{j}\right)
$$

$A L_{p}$ is the average length of coupling paths in the network, $d\left(v_{i}, v_{j}\right)$ is the distance between node $i$ and node $j$, and $n$ is the number of nodes in the coupling network.

\section{Empirical research}

\subsection{Conditions in Dalian}

Dalian is the core city in the coastal area of North China. It is in the highlands, which form a banded group structure surrounded by natural mountains. Dalian has retained urban morphology from its Russian and Japanese colonial periods. This is an urban morphology that combines the Baroque style with crosscutting axes and chessboard styles. This allows for abundant and diverse empirical research materials. As it is shown in Figure 1 - Figure 5, we developed a research sequence extending outward to the urban fringe from around the urban historic area. This formed a group of step transitions which match the urban morphological and functional characteristics. 


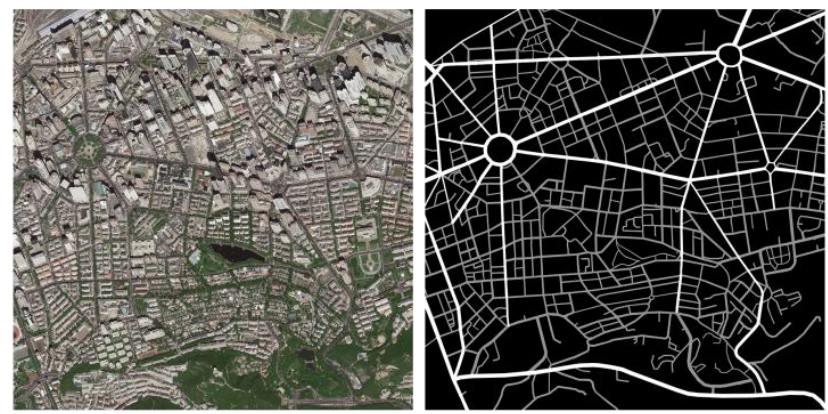

Figure 1. The historical district sample (Gangwan Square Areas).
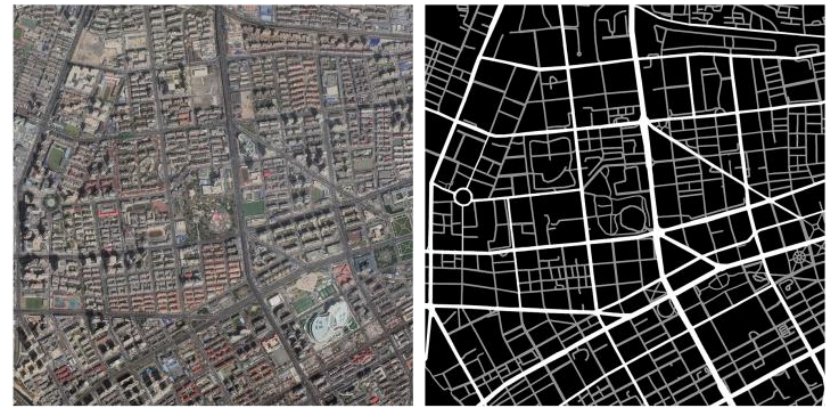

Figure 2. The central urban district sample (Zhongshan Park).

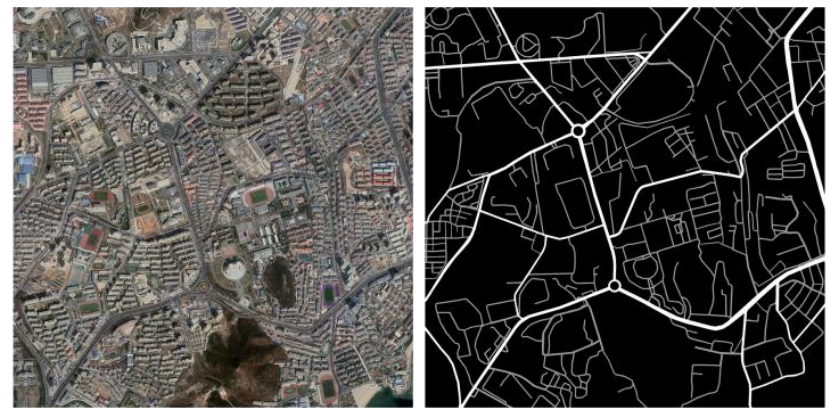

Figure 3. The new urban district sample (Heishijiao Areas).
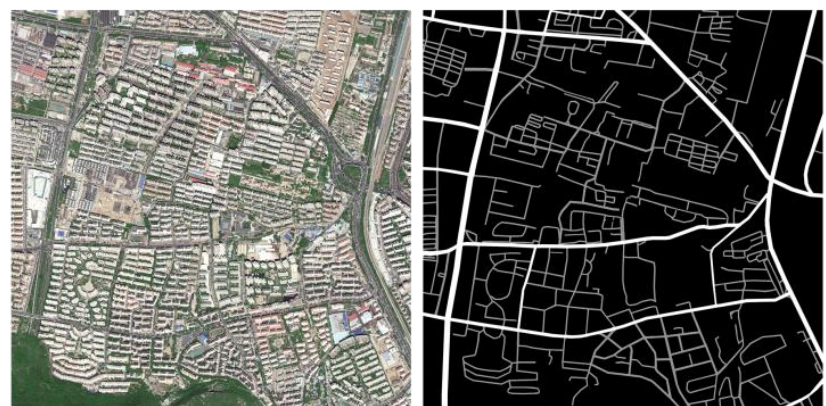
Figure 4. The urban fringe district sample (Wanda Square).

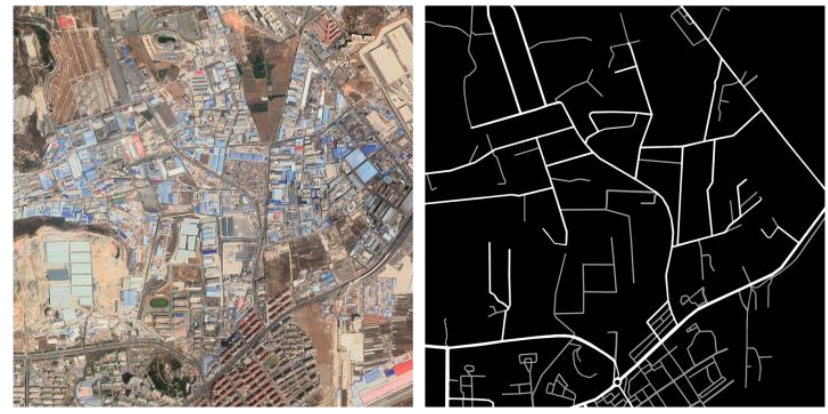

Figure 5. The suburban district sample (North Railway Station Areas).

\subsection{Charts}

The five blocks cover the different street morphologies and function types. We adopted time limits for pedestrians (i.e., $20 \mathrm{~min}$, or 1,200 m) as the basis for defining the sample borders of the blocks. This, together with the research range of each sample at 2,400 $\mathrm{m} \times 2,400 \mathrm{~m}$ rectangle, showed a double1,200. As shown in Figures 2 to 6 , we performed a transverse analysis on the same sample at different scale levels. We also performed a longitudinal analysis of different land uses.

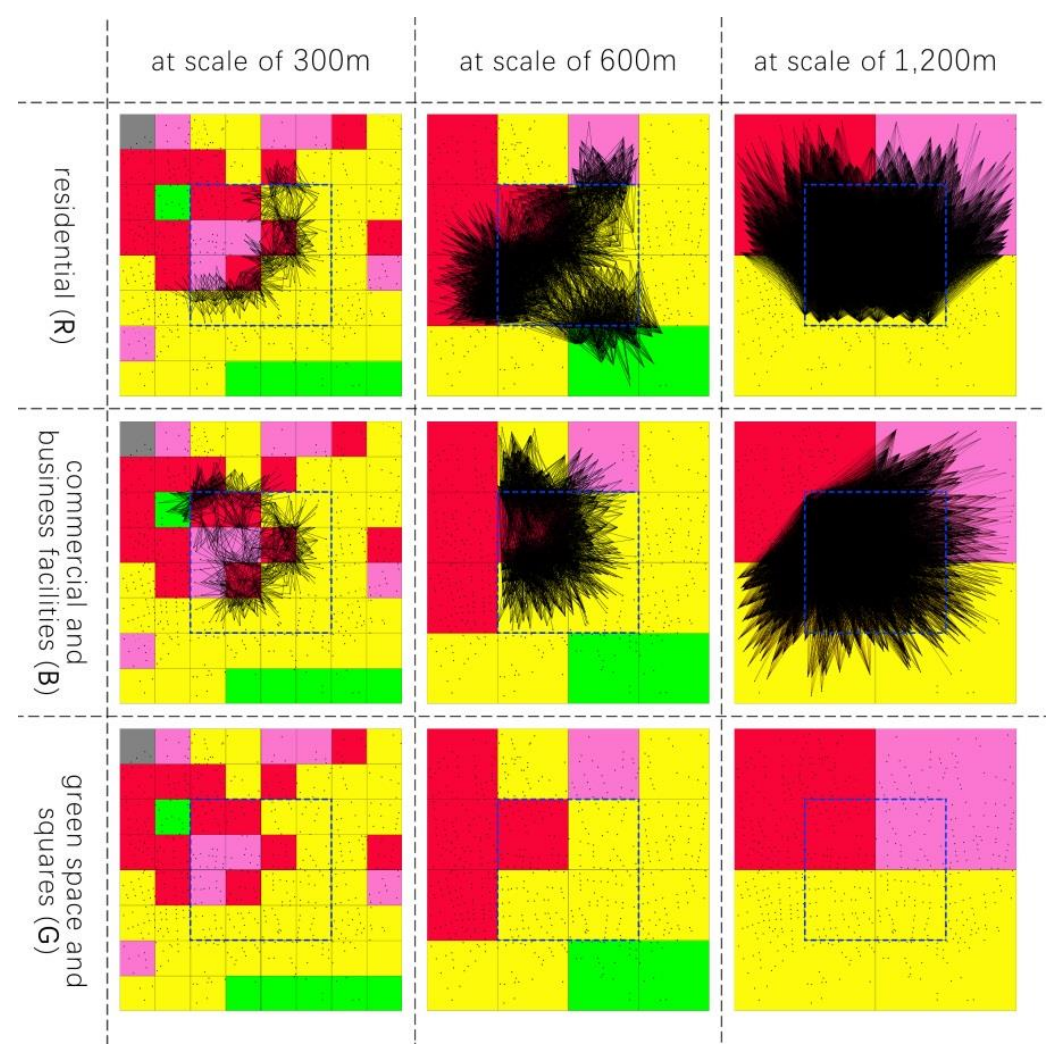

Figures 6 coupling network of the historical district sample 


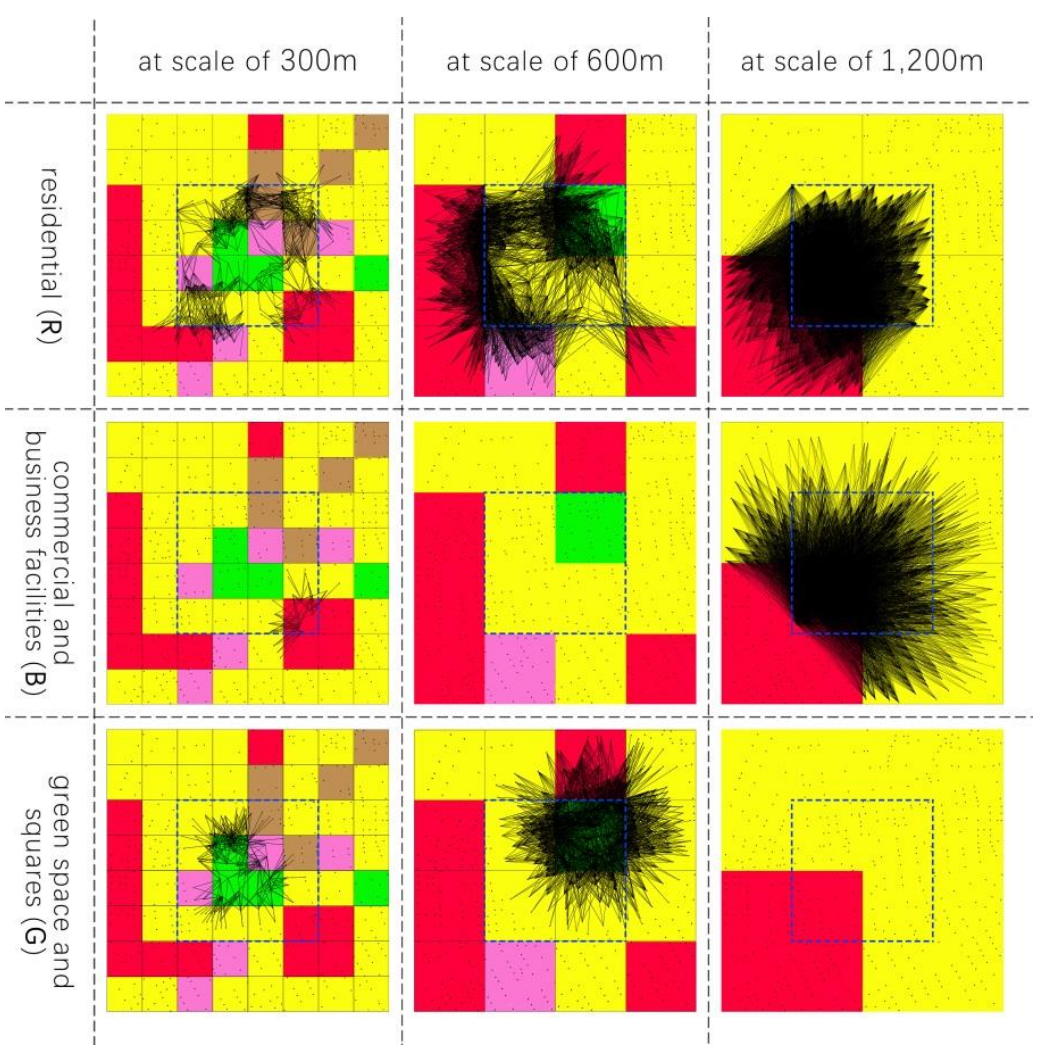

Figures 7 coupling network of the central urban district sample

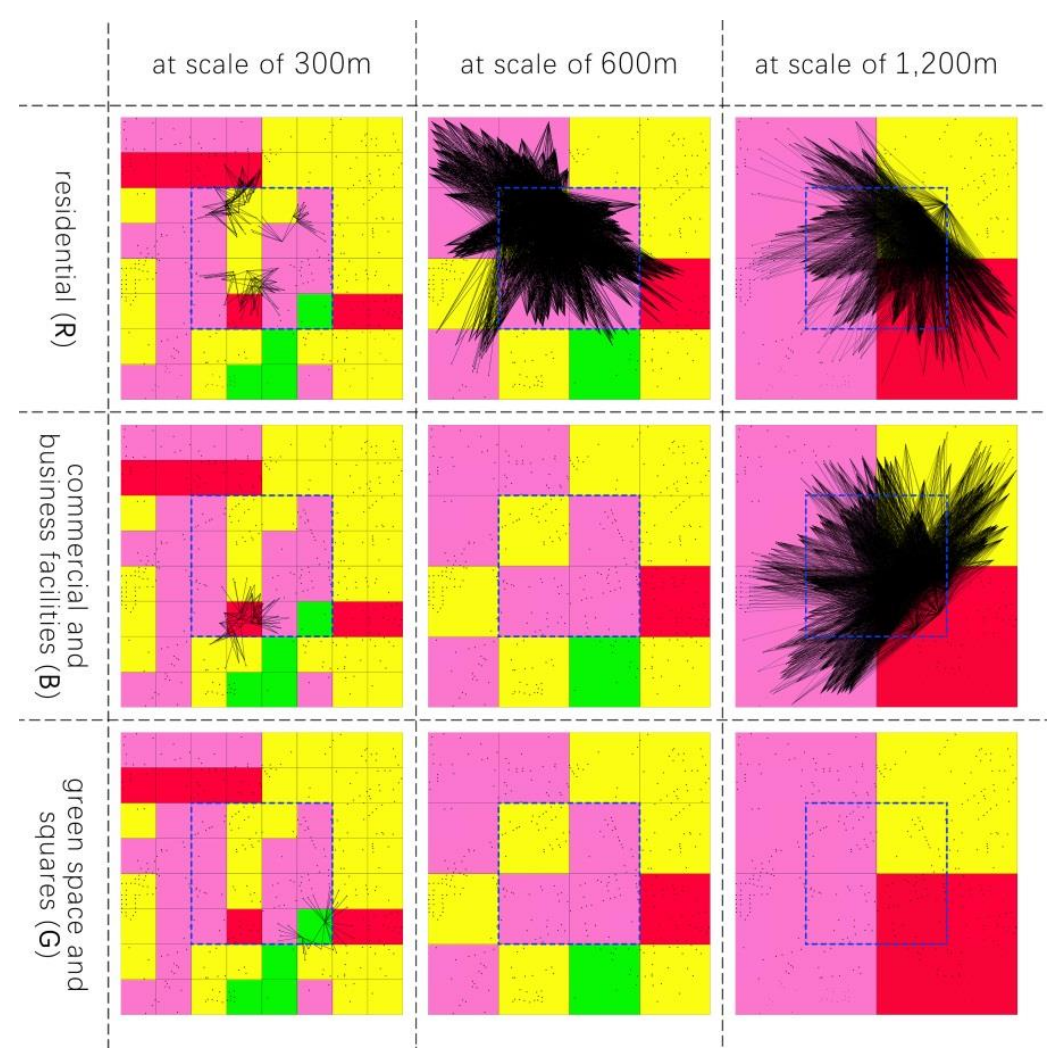

Figures 8 coupling network of the new urban district sample 


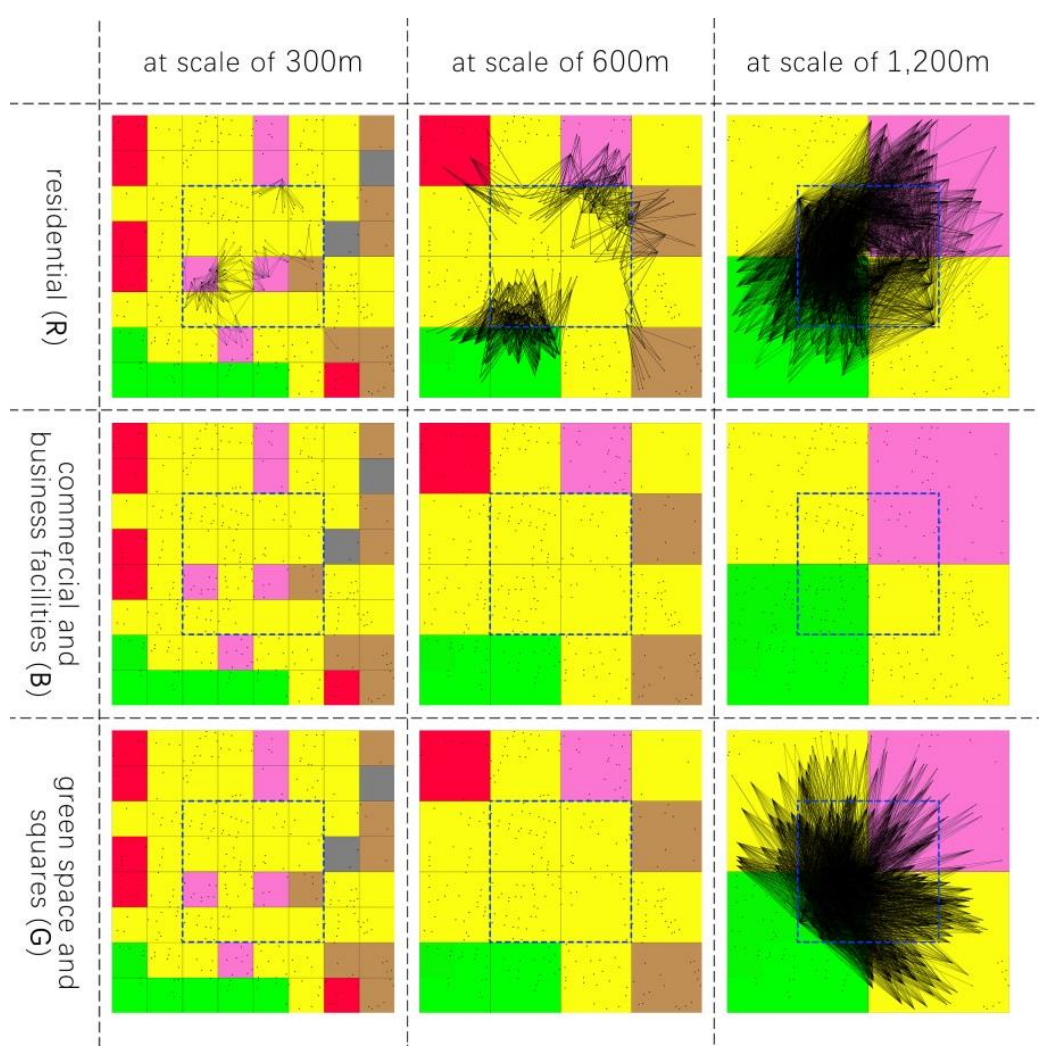

Figures 9 coupling network of the urban fringe district

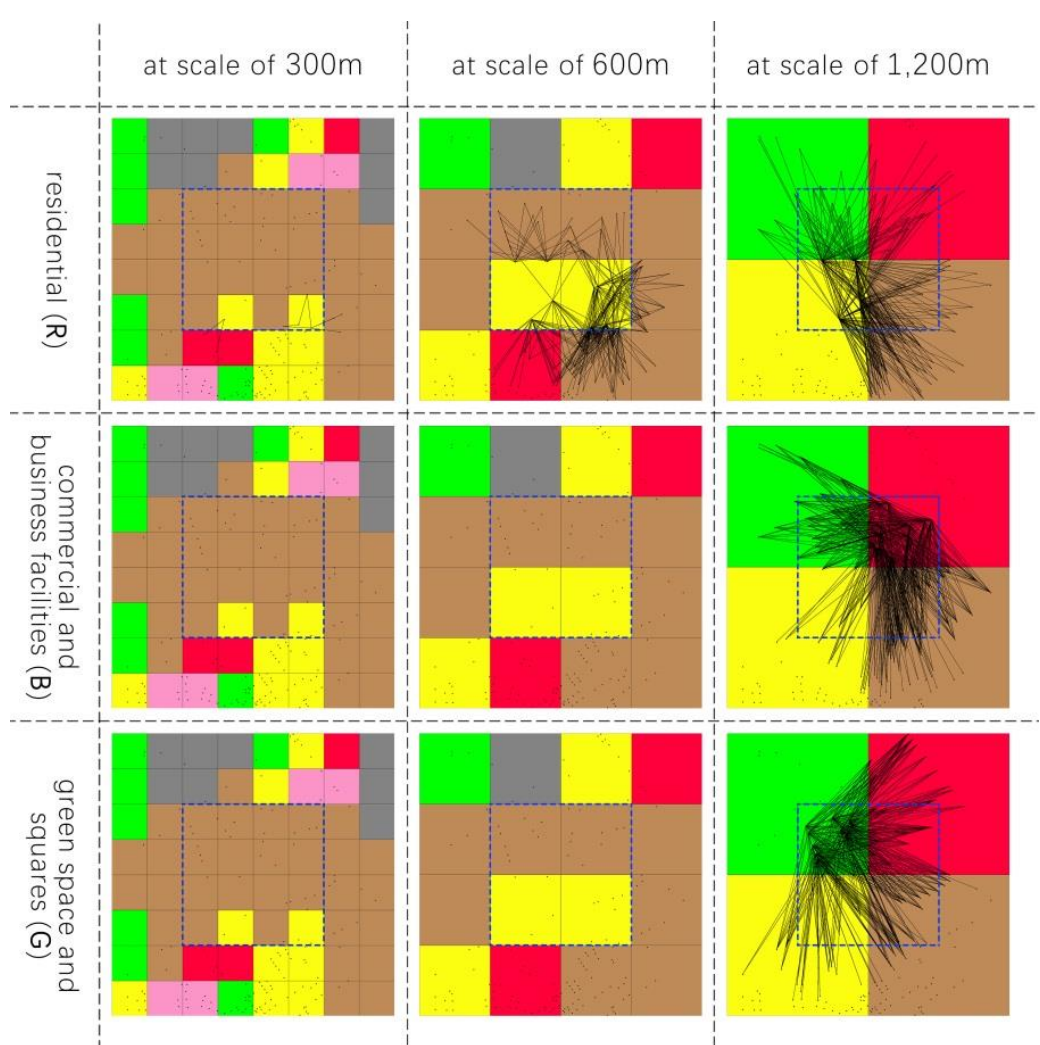

Figures 10 coupling network of the the suburban district sample 
We calculated parameters for the coupling clustering coefficient, average coupling clustering coefficient, total coupling path length and average coupling path length in all samples. We list them in Table 2- Table 5.

Table 2. Coupling clustering coefficient.

\begin{tabular}{|c|c|c|c|c|}
\hline land use & at scale of $300 \mathrm{~m}$ & at scale of $600 \mathrm{~m}$ & at scale of $1,200 \mathrm{~m}$ & Total \\
\hline & \multicolumn{4}{|c|}{ CCC of the historical district sample (Gangwan Square Areas) } \\
\hline $\mathrm{R}$ & 1.29 & 1.52 & 1.89 & 4.70 \\
\hline B & 1.27 & 1.63 & 1.83 & 4.73 \\
\hline \multirow[t]{2}{*}{$\bar{G}$} & 0.00 & 0.00 & 0.00 & 0.00 \\
\hline & \multicolumn{4}{|c|}{ CCC of the central urban district sample(Zhongshan Park) } \\
\hline $\mathrm{R}$ & 1.20 & 1.46 & 1.90 & 4.56 \\
\hline B & 1.22 & 0.00 & 1.85 & 3.07 \\
\hline \multirow[t]{2}{*}{ G } & 1.23 & 1.69 & 0.00 & 2.92 \\
\hline & \multicolumn{4}{|c|}{ "CC of the new urban district sample (Heishijiao Areas) } \\
\hline $\mathrm{R}$ & 1.19 & 1.45 & 1.68 & 4.23 \\
\hline B & 1.20 & 0.00 & 1.62 & 2.82 \\
\hline \multirow[t]{2}{*}{ G } & 1.15 & 0.00 & 0.00 & 1.15 \\
\hline & \multicolumn{4}{|c|}{ CCC of the urban fringe district sample (Wanda Square) } \\
\hline $\mathrm{R}$ & 1.08 & 1.10 & 1.67 & 3.85 \\
\hline$B$ & 0.00 & 0.0 & 0.00 & 0.00 \\
\hline \multirow[t]{2}{*}{ G } & 0.00 & 0.00 & 1.76 & 1.76 \\
\hline & \multicolumn{4}{|c|}{ CC of the suburban district sample (North Railway Station Areas) } \\
\hline $\mathrm{R}$ & 1.14 & 1.36 & 1.58 & 4.08 \\
\hline B & 0.00 & 0.00 & 1.66 & 1.66 \\
\hline G & 0.00 & 0.00 & 1.60 & 1.60 \\
\hline
\end{tabular}

Table 3. Average coupling clustering coefficient.

\begin{tabular}{ccccc}
\hline \hline land use & at scale of $300 \mathrm{~m}$ & at scale of $600 \mathrm{~m}$ & at scale of $1,200 \mathrm{~m}$ & Total \\
\hline \hline $\mathrm{R}$ & \multicolumn{2}{c}{ ACC of the historical district sample (Gangwan Square Areas) } & \\
\hline \hline $\mathrm{B}$ & 0.63 & 0.74 & 0.85 & 2.22 \\
\hline \hline
\end{tabular}


Quantitative Analysis of the Topologic

Xiao, Y.; Wang, B.; Sun, H.

\begin{tabular}{|c|c|c|c|c|}
\hline land use & at scale of $300 \mathrm{~m}$ & at scale of $600 \mathrm{~m}$ & at scale of $1,200 \mathrm{~m}$ & Total \\
\hline G & 0.00 & 0.00 & 0.00 & 0.00 \\
\hline \multicolumn{5}{|c|}{ ACC of the central urban district (Zhongshan Park) } \\
\hline $\mathrm{R}$ & 0.62 & 0.70 & 0.81 & 2.13 \\
\hline B & 0.49 & 0.00 & 0.76 & 1.25 \\
\hline G & 0.52 & 0.75 & 0.00 & 1.27 \\
\hline \multicolumn{5}{|c|}{ ACC of the new urban district sample (Heishijiao Areas) } \\
\hline $\mathrm{R}$ & 0.60 & 0.67 & 0.79 & 2.06 \\
\hline B & 0.43 & 0.00 & 0.70 & 1.13 \\
\hline $\mathrm{G}$ & 0.00 & 0.70 & 0.73 & 1.43 \\
\hline
\end{tabular}

ACC of the urban fringe district (Wanda Square)

\begin{tabular}{ccccc}
\hline \hline $\mathrm{R}$ & 0.52 & 0.60 & 0.70 & 1.82 \\
\hline \hline $\mathrm{B}$ & 0.00 & 0.00 & 0.00 & 0 \\
\hline \hline $\mathrm{G}$ & 0.00 & 0.00 & 0.78 & 0.78 \\
\hline \hline
\end{tabular}

ACC of the suburban district sample (North Railway Station Areas)

\begin{tabular}{ccccc}
\hline \hline $\mathrm{R}$ & 0.26 & 0.66 & 0.65 & 1.57 \\
\hline \hline $\mathrm{B}$ & 0.40 & 0.00 & 0.64 & 1.04 \\
\hline \hline $\mathrm{G}$ & 0.00 & 0.00 & 0.77 & 0.77
\end{tabular}

Table 4. Total coupling path length.

\begin{tabular}{|c|c|c|c|c|}
\hline land use & at scale of $300 \mathrm{~m}$ & at scale of $600 \mathrm{~m}$ & at scale of $1,200 \mathrm{~m}$ & Total \\
\hline & \multicolumn{4}{|c|}{$L_{p}$ of the historical district sample (Gangwan Square Areas) (km) } \\
\hline $\mathrm{R}$ & 152.08 & 2299.45 & 15926.46 & 18377.99 \\
\hline $\mathrm{B}$ & 298.08 & 1107.77 & 9465.83 & 10871.38 \\
\hline \multirow[t]{2}{*}{ G } & 0.00 & 0.00 & 0.00 & 0.00 \\
\hline & \multicolumn{4}{|c|}{$L_{p}$ of the central urban district (Zhongshan Park) (km) } \\
\hline $\mathrm{R}$ & 246.09 & 1807.47 & 6399.67 & 8453.23 \\
\hline$B$ & 6.56 & 0.00 & 5705.33 & 5711.89 \\
\hline \multirow[t]{2}{*}{ G } & 16.25 & 108.04 & 0.00 & 124.29 \\
\hline & \multicolumn{4}{|c|}{$L_{p}$ of the new urban district sample (Heishijiao Areas) $(\mathrm{km})$} \\
\hline $\mathrm{R}$ & 13.41 & 974.88 & 3600.89 & 4589.18 \\
\hline
\end{tabular}


Quantitative Analysis of the Topologic

Xiao, Y.; Wang, B.; Sun, H.

\begin{tabular}{|c|c|c|c|c|}
\hline land use & at scale of $300 \mathrm{~m}$ & at scale of $600 \mathrm{~m}$ & at scale of $1,200 \mathrm{~m}$ & Total \\
\hline B & 6.68 & 0.00 & 5459.67 & 5466.35 \\
\hline \multirow[t]{2}{*}{ G } & 8.42 & 0.00 & 0.00 & 8.42 \\
\hline & \multicolumn{4}{|c|}{$L_{p}$ of the urban fringe district (Wanda Square) $(\mathrm{km})$} \\
\hline R & 62.68 & 292.03 & 4567.36 & 4922.07 \\
\hline B & 0.00 & 0.00 & 0.00 & 0.00 \\
\hline \multirow[t]{2}{*}{ G G } & 0.00 & 0.00 & 3879.95 & 3879.95 \\
\hline & \multicolumn{4}{|c|}{$L_{p}$ of the suburban district sample (North Railway Station Areas) (km) } \\
\hline R & 2.39 & 257.01 & 278.34 & 537.74 \\
\hline B & 0.00 & 0.00 & 452.48 & 452.48 \\
\hline G & 0.00 & 0.00 & 361.01 & 361.01 \\
\hline
\end{tabular}

Table 5. Average coupling path length.

\begin{tabular}{|c|c|c|c|c|}
\hline land use & at scale of $300 \mathrm{~m}$ & at scale of $600 \mathrm{~m}$ & at scale of $1,200 \mathrm{~m}$ & Total \\
\hline & \multicolumn{4}{|c|}{$A L_{p}$ of the historical district sample (Gangwan Square Areas) (m) } \\
\hline $\mathrm{R}$ & 2.32 & 15.73 & 54.45 & 72.50 \\
\hline$B$ & 1.60 & 5.96 & 55.41 & 62.97 \\
\hline \multirow[t]{2}{*}{ G } & 0.00 & 0.00 & 0.00 & 0.00 \\
\hline & \multicolumn{4}{|c|}{$A L_{p}$ of the central urban district (Zhongshan Park) (m) } \\
\hline $\mathrm{R}$ & 2.16 & 13.85 & 46.14 & 62.15 \\
\hline$B$ & 0.06 & 0.00 & 50.04 & 50.10 \\
\hline \multirow[t]{2}{*}{ G } & 0.14 & 0.95 & 0.00 & 1.09 \\
\hline & \multicolumn{4}{|c|}{$\bar{A} A L_{p}$ of the new urban district sample (Heishijiao Areas) (m) } \\
\hline $\mathrm{R}$ & 0.17 & 12.66 & 36.75 & 49.58 \\
\hline B & 0.09 & 0.00 & 30.88 & 30.97 \\
\hline \multirow[t]{2}{*}{ G } & 0.11 & 0.00 & 0.00 & 0.11 \\
\hline & \multicolumn{4}{|c|}{$A L_{p}$ of the urban fringe district (Wanda Square) (m) } \\
\hline $\mathrm{R}$ & 1.33 & 6.18 & 22.61 & 30.12 \\
\hline B & 0.00 & 0.00 & 0.00 & 0.00 \\
\hline $\mathrm{G}$ & 0.00 & 0.00 & 29.07 & 29.07 \\
\hline
\end{tabular}

$A L_{p}$ of the suburban district sample (North Railway Station Areas) (m) 


\begin{tabular}{|c|c|c|c|c|}
\hline land use & at scale of $300 \mathrm{~m}$ & at scale of $600 \mathrm{~m}$ & at scale of $1,200 \mathrm{~m}$ & Total \\
\hline $\bar{R}$ & 0.20 & 11.82 & 36.63 & 48.65 \\
\hline B & 0.00 & 0.00 & 40.41 & 40.41 \\
\hline G & 0.00 & 0.00 & 30.64 & 30.64 \\
\hline
\end{tabular}

\section{Analysis}

\subsection{Connectivity analysis of the coupling network of block samples}

The historical district sample is a region with a long history. Its spatial locale adopts the Baroque axis network style, in which there are characteristics of both a chessboard and cross-cutting street patterns. This challenge is alleviated by the inconvenient transport of diagonal connections through traditional checkerboard layouts. Thus, the average coupling clustering coefficient of residential $(R)$ and commercial and business facilities (B) of the historical district sample is higher than that of the central urban district sample. Due to the scattered green spaces, the average coupling clustering coefficient of green spaces and public squares $(G)$ of the central urban district sample on a small scale is higher than that of the other sample.

The new urban district sample is a combination of different street morphological types due to its structural characteristics in tree-like and chessboard patterns. The new urban district has a large number of residential areas, and its hierarchical classification of internal roads is unclear. Furthermore, continuous streets and collocated interface functions along the streets in residential areas contribute to the concentration of diversity in space and connection of residential (R).

The central urban district sample shows small blocks, uniform space, and high density. A horizontal comparison of sample data also shows that the central urban district sample has a high average coupling clustering coefficient at large scale. This consists of many loops, with many intersections and grid-like street units. The coupling clustering coefficient values for the the central urban district sample falls between the new urban district sample and the historical district sample. In general, the data show a moderate network coupling connectivity.

The road layout of the urban fringe district and the suburban district includes multilevel road branches, reflecting the characteristics of a tree-like street pattern. This street pattern affects and limits the fringe district sample. Thus their spatial connectivities are weak, and the average coupling clustering coefficient values are low, with scattered urban development lands.

Based on comparison of the historical district, the central urban district, the fringe district, and the suburban district, the results show significant differences in average coupling clustering coefficient for commercial and business facilities land (B). These differences reflect a lack of diversity in commercial, leisure, recreational, and cultural facilities for the fringe district and the suburban district. Meanwhile, lowered attractiveness covered small areas within the entire sample. But the coupling clustering coefficient value of the fringe district sample and the suburban district sample, for green space and squares $(G)$, is higher than the other samples. In general, the results of differenr samples show that there have been improvements to the urban and rural greening integration in Dalian. 


\subsection{Accessibility analysis of coupling networks for block samples}

The accessibility of a coupling network reflects the average coupling path length of each node to the surrounding in built environment. The central urban district has small-scale blocks and high-density road networks. Thus it supplies flexible and varied route options. As a result, most nodes have higher topological accessibility than in other samples, thus revealing strong characteristics of topological accessibility. The difference is quite clear in the total value of coupling path length between the central urban district and the suburban district, especially in a large scale. Besides, the streets in the historical district sample are short and tortuous. This results in a clear distribution of coupling path length for the spatial network. The twists and turns in the internal topological morphology of the sample, and its tortuous street space across walking distance affect, to a certain extent, the attraction of the area for personal walks and gatherings.

The central urban district sample has many large shopping malls and features catering to retail stores along the street. It has a large pedestrian flow and traffic stream in a large scale, showing that it offers a high degree of accessibility for its surrounding built environment. The average coupling path length of the urban fringe district, for residential $(R)$ at mesoscale, is obviously lower than that in the central urban district. This is because the traffic that flows in a one-way manner between different topological levels in the urban fringe district sample destroys the horizontal accessibility and overlap of spatial functions and street activities. Thus, the section lacks a core functional area with strong advantages. This reflects the average coupling path length and reveals the network accessibility of spatial samples at distinct locations and under various built enviroment. It also illustrates the attractiveness, for individuals' walking activities and potential gatherings, of the different functional attributes of land uses.

\section{Conclusions}

We applied a grid-coupled network analysis to five local block samples in various built environments. Due to the complex and changeable nature of streets' spatial morphology in bulit environments, we built a model for spatial coupling analysis. We compared and analyzed the samples' coupling connectivity and accessibility via quantitative research methods.

Our findings reveal that there are many similarities and differences in urban street form and function. Furthermore, the limiting factors of our samples influence the horizontal and vertical comparisons of different street form, block function analyses, and street vitality. Additionally, various planning measures can affect street spaces per the topological forms and function structures. This is possible by verifying the accessibility and connectivity of the spatial coupling networks for distinct types of urban blocks in urban bulit environments. Our study thus verifies the effectiveness and applicability of the coupling network model, based on empirical research and case analyses. Given the complexity of built enviroment, modeling methods and personal cognitive capabilities have inherent limitations. There are still inevitable technical problems and limitations associated with using spatial coupling analysis models. But such models still have high application prospects. They can reflect the spatial topological features of different blocks and streets in different stages of urban development across different historical periods. They also supply evaluations of the coupling coordination degree of urban form and function.

The coupling analysis model presented here can apply to land use planning in China's urban planning system. The coupling analysis model will be useful for analyzing the relationship between the function layout of land use and individuals' behavior and activities in urban spaces. Future studies, by proposing a spatial optimization strategy that helps in urban design and decision analysis, should strengthen the integration of urban topology and functional structures. This may lead to practical planning and design efforts. In summary, this study supplies a tool for urban designers and researchers to use in analyzing and 
assessing urban spatial and functional structures. This method supplies effective feedback on the requirements of urban spatial systems. And it does so in keeping with the guidelines of sustainable urban design.

\section{References}

Batty, M. \& Longley, P. A. (1994) Fractal cities: a geometry of form and function. 1rd edn. Pittsburgh: Academic Press.

Bibri, S. E., Krogstie, J. J. S. 'ICT of the new wave of computing for sustainable urban forms: Their big data and context-aware augmented typologies and design concepts', C. \& Society, 32, p449-474.

Cardillo, A., Scellato, S., Latora, V. \& Porta, S. (2006) 'Structural properties of planar graphs of urban street patterns', J. P. R. E., 73(6), p66-71.

Chan, S. H. Y.; Donner, R. V.; Laemmer, S. (2011) 'Urban road networks: Spatial networks with universal geometric features? A case study on Germany's largest cities', Eur. Phys. J. B., 84(4), p563-577.

Gastner, M.T.; Newman, M.E.J. (2006) 'The spatial structure of networks', T. E. P. J. B.-C. M. \& Systems, C., 49(2), p247-252.

Hacar, M. (2020) 'A rule-based approach for generating urban footprint maps: from road network to urban footprint', J. Eng. Geosci, 5(2), p100-108.

Hillier, B.; Hanson, J. (1984) The Social Logic of Space. London: Cambridge University Press.

Hillier, B. (2008) 'Space and spatiality: what the built environment needs from social theory', Build. Research Inf., 36(3), pp216-30.

Jacobs, A. (1993) 'Great streets', B. J. A. M., 1(3), P23-27.

Jiang, B., Claramunt, C. J. E., (2004) 'Topological analysis of urban street networks', Planning, P. B. \& design, 31(1), p151-162.

Lämmer, S., Gehlsen, B., Helbing, D. (2006) 'Scaling laws in the spatial structure of urban road networks', J. P. A. S. M. \& Applications, 363(1), p89-95.

Lynch, K. (1984) Reconsidering the Image of the City. New York: Springer.

Marshall, S. (2004) Streets and patterns. London: Routledge.

Marshall, S., Gil, J., Kropf, K., Tomko, M., Figueiredo, L. (2018) 'Street network studies: from networks to models and their representations', J. N. \& Economics, S., 18(3), pp735-749.

Ravasz, E. \& Barabási, A.-L. (2003) Hierarchical organization in complex networks, J. P. r. E., 67(2), 026112.

Salingaros, N. A. (2005) Principles of urban structure. Netherlands: Techne Press.

Salingaros, N. A. (1998) 'Theory of the urban web', J. Urban Design, 3(1), pp53-71.

Scellato, S.; Cardillo, A.; Latora, V.; Porta, S. (2006) 'The backbone of a city', Eur. Phys. J. B, 50(1-2), pp221-225.

Sharif, A. (2019) 'Resilient urban forms: A review of literature on streets and street networks', Build. Environ, 147, pp171-87.

Stratford, E.; Waitt, G.; Harada, T. (2020) 'Walking city streets: Spatial qualities, spatial justice, and democratising impulses', Trans. Inst. Br. Geogr., 45(1), pp123-138. 
Tang, L., Lin, Y., Li, S., Li, S., Li, J., Ren, F. \& Wu, C. (2018) 'Exploring the influence of urban form on urban vibrancy in Shenzhen based on mobile phone data', Sustainability, 10(12), 4565.

Thomas, R.; Fordha, M. (2003) Sustainable Urban Design: An Environmental Approach. London: Spon Press.

Wang, S.; Yu, D.; Kwan, M. P.; Zhou, H.; Li, Y.; Miao, H. (2019) 'The evolution and growth patterns of the road network in a medium-sized developing city: A historical investigation of Changchun, China, from 1912 to 2017'. Sustainability, 11(19), 5307.

Xie, F., Levinson, D. (2007) 'Measuring the structure of road networks', Geogr. Anal. 39(3), pp336-356.

Yue, H.; Zhu, X. (2019) 'Exploring the relationship between urban vitality and street centrality based on social network review data in Wuhan, China', Sustainability, 11(16), 4356. 